\title{
Unambiguous Quantum State Discrimination and the Equal Probability Measurement
}

\author{
Yonina C. Eldar \\ Department of Electrical Engineering \\ Technion-Israel Institute of Technology \\ Technion City, Haifa 32000, Israel \\ e-mail: yonina@ee.technion.ac.il
}

\section{INTRODUCTION}

In a quantum detection problem a transmitter conveys classical information to a receiver using a quantum-mechanical channel. We assume that each message corresponds to a pure quantum state $\left\{\left|\phi_{i}\right\rangle, 1 \leq i \leq m\right\}$. The information is detected by subjecting the system to a quantum measurement, optimized to distinguish between pure nonorthogonal states.

In unambiguous quantum detection [1] a measurement is designed comprising $m+1$ operators $\left\{\Pi_{i}, 0 \leq i \leq m\right\}$ satisfying $\sum_{i=0}^{m} \Pi_{i}=I$, that with a certain probability returns an inconclusive result, but such that if the measurement returns an answer, then the answer is correct with probability one. Each operator $\Pi_{i}, 1 \leq i \leq m$ corresponds to detection of the corresponding state $\left|\phi_{i}\right\rangle, 1 \leq i \leq m$, and $\Pi_{0}$ corresponds to an inconclusive result.

An unambiguous measurement exists if and only if the states are linearly independent [2]. In this case, without loss of generality [3], $\Pi_{i}=p_{i}\left|\tilde{\phi}_{i}\right\rangle\left\langle\tilde{\phi}_{i}\right| \triangleq p_{i} Q_{i}, 1 \leq i \leq m$, for some $p_{i} \geq 0$, where $\left|\tilde{\phi}_{i}\right\rangle \in \mathcal{U}$ are the unique vectors in the space $\mathcal{U}$ spanned by the vectors $\left|\phi_{i}\right\rangle$ satisfying $\left\langle\tilde{\phi}_{i} \mid \phi_{k}\right\rangle=\delta_{i k}, 1 \leq i, k \leq$ $m$. If the state $\left|\phi_{i}\right\rangle$ is prepared with prior probability $\eta_{i}$, then the total probability of correctly detecting the state is

$$
P_{D}=\sum_{i=1}^{m} \eta_{i}\left\langle\phi_{i}\left|\Pi_{i}\right| \phi_{i}\right\rangle=\sum_{i=1}^{m} \eta_{i} p_{i} .
$$

The problem then is to choose $p_{i} \geq 0$ to maximize $P_{D}$ subject to $\sum_{i=1}^{m} p_{i} Q_{i} \leq I$.

\section{Optimal Detection And the EPM}

As we show in [3], the problem of (1) can be formulated as a convex semidefinite programming problem (SDP) [4]. By exploiting the many well known algorithms for solving SDPs, which are guaranteed to converge to the global optimum, the optimal measurement can be computed very efficiently in polynomial time. Furthermore, the SDP formulation can be used to derive necessary and sufficient conditions for optimality on $p_{i}$, as incorporated in the following theorem.

Theorem 1 Let $\Lambda$ denote the scalars $p_{i} \geq 0,1 \leq i \leq m$ that satisfy $\sum_{i=1}^{m} p_{i} Q_{i} \leq I$, and let $\Gamma$ denote the matrices $X \geq 0$ and scalars $z_{i} \geq 0,1 \leq i \leq m$ such that $\operatorname{Tr}\left(Q_{i} X\right)-z_{i}=\eta_{i}$. Then $p_{i} \in \Lambda$ maximizes $P_{D}$ if and only if there exists $X, z_{i} \in \Gamma$ such that $X\left(I-\sum_{i=1}^{m} p_{i} Q_{i}\right)=0$ and $z_{i} p_{i}=0,1 \leq i \leq m$.

A simple suboptimal measurement for unambiguous discrimination is the equal-probability measurement (EPM) in which $p_{i}=p, 1 \leq i \leq m$. Using Theorem 1 we can derive conditions under which the EPM is optimal. In particular, we must have that $p=\sigma_{m}^{2}$ where $\sigma_{m}$ is the smallest singular value of the matrix $\Phi$ of columns $\left|\phi_{i}\right\rangle$. In addition, we have the following theorem.
Theorem 2 Let $\Phi$ have an $S V D \Phi=U \Sigma V^{*}$, let $\left|v_{i}\right\rangle$ denote the columns of $V^{*}$ and $v_{i}(k)$ the kth component of $\left|v_{i}\right\rangle$, and let $s$ be the multiplicity of the smallest singular value $\sigma_{m}$. Then,

1. If $s=1$ then the EPM is optimal if and only if $\left|v_{i}(m)\right|^{2}=\eta_{i}$ for $1 \leq i \leq m$;

2. If $s>1$ then the EPM is optimal if there exists $b_{i} \geq$ $0,1 \leq i \leq s$ such that $A b=\eta$ where $b$ and $\eta$ are the vectors of components $b_{i}$ and $\eta_{i}$ respectively, and

$$
A=\left[\begin{array}{ccc}
\left|v_{1}(m)\right|^{2} & \cdots & \left|v_{1}(m-s+1)\right|^{2} \\
\left|v_{2}(m)\right|^{2} & \cdots & \left|v_{2}(m-s+1)\right|^{2} \\
\vdots & & \vdots \\
\left|v_{m}(m)\right|^{2} & \cdots & \left|v_{m}(m-s+1)\right|^{2}
\end{array}\right] ;
$$

3. For an arbitrary state set, if we choose the prior probabilities $\eta=A b$ where $b_{i} \geq 0$ are arbitrary coefficients satisfying $\sum_{i=1}^{m} b_{i}=1$, then the EPM is optimal;

4. If $\left\langle\phi_{i}\left|\left(\Phi \Phi^{*}\right)^{t / 2-1}\right| \phi_{i}\right\rangle=\eta_{i} a_{t}, 1 \leq i \leq m, 1 \leq t \leq m$ for some constants $a_{t}$, then the EPM is optimal.

Chefles and Barnett [5] showed that the EPM is optimal in the special case in which the state vectors form a cyclic set. Using Theorem 2 we can generalize this result to a large class of state sets. Specifically, for geometrically uniform state sets $\mathcal{S}=\left\{\left|\phi_{i}\right\rangle=U_{i}|\phi\rangle, U_{i} \in \mathcal{G}\right\}$ where $\mathcal{G}$ is a group of unitary matrices, the EPM is optimal. For compound geometrically uniform (CGU) state sets $\mathcal{S}=\left\{\left|\phi_{i k}\right\rangle=U_{i}\left|\phi_{k}\right\rangle, 1 \leq i \leq l, 1 \leq\right.$ $k \leq r\}$ where the matrices $\left\{U_{i}, 1 \leq i \leq l\right\}$ are unitary and form a group $\mathcal{G}$, and the vectors $\left\{\left|\phi_{k}\right\rangle, 1 \leq k \leq r\right\}$ are the generating vectors, the EPM is optimal if

$$
\left\langle\phi_{k}\left|\left(\Phi \Phi^{*}\right)^{t / 2-1}\right| \phi_{k}\right\rangle=a_{t}, \quad 1 \leq k \leq r, 1 \leq t \leq m,
$$

for some constants $a_{t}$. In particular, if the generating vectors $\left\{\left|\phi_{k}\right\rangle=V_{k}|\phi\rangle, 1 \leq k \leq r\right\}$ are themselves GU, and $U_{p} V_{t}=$ $V_{t} U_{p} e^{j \theta(p, t)}$ for all $t$ and $p$ and arbitrary $\theta(p, t)$, then the EPM is optimal.

\section{REFERENCES}

[1] I. D. Ivanovic, "How to differentiate between non-orthogonal states," Phys. Lett. A, vol. 123, pp. 257-259, Aug. 1987.

[2] A. Chefles, "Unambiguous discrimination between linearly independent quantum states," Phys. Lett. A, vol. 239, pp. 339347, Apr. 1998.

[3] Y. C. Eldar, "A semidefinite programming approach to optimal unambiguous discrimination of quantum states," IEEE Trans. Inform. Theory, vol. 49, pp. 446-456, Feb. 2003.

[4] L. Vandenberghe and S. Boyd, "Semidefinite programming," SIAM Rev., vol. 38, no. 1, pp. 40-95, Mar. 1996.

[5] A. Chefles and S. M. Barnett, "Optimum unambiguous discrimination between linearly independent symmetric states," Phys. Lett. A, vol. 250, pp. 223-229, 1998. 